\title{
ЗАЛЕЖНІСТЬ ПЕРЕРІЗУ РОЗСІЯННЯ СВІТЛА МЕТАЛЕВИМИ НАНОЧАСТИНКАМИ ВІД ЇХ ФОРМИ
}

\author{
П.М. ТОМЧУК
}

УдК 53

Інститут фізики НАН України, відділ теоретичної фізики

(Просn. Науки, 46, Київ 03680; e-mail: ptomchuk@iop. kiev. ua)

\begin{abstract}
Розвинуто теорію розсіяння світла еліпсоїдальними металевими наночастинками, розміри яких менші за довжину вільного пробігу електрона у частинці, а також значно менші за довжину електромагнітної хвилі. Враховано вплив форми наночастинки як на частоти плазмових резонансів, так і на їх півширини. У задачі розсіяння світла вперше враховано тензорний характер оптичної провідності, що наявний у випадку таких розмірів еліпсоподібних металевих наночастинок. За допомогою отриманого виразу для поперечника розсіяння проілюстровано істотний вплив форми частинки на частотні і поляризаційні залежності розсіяного світла.
\end{abstract}

\section{1. Вступ}

Починаючи з класичних результатів Релея (1871р.) явищу розсіяння світла малими частинками, розмір яких значно менший за довжину розсіючої хвилі, присвячено велику кількість теоретичних і експериментальних робіт. Огляд основних результатів, отриманих у цьому напрямку, можна знайти у недавно опублікованих монографіях [1, 2] і цитованій там літературі. Згідно з теорією Релея переріз розсіяння світла сферичною частинкою на далекій відстані від неї пропорційний $\omega^{4}|\alpha(\omega)|^{2}$. Тут $\omega$ - частота світла, $\alpha(\omega)$ - поляризованість частинки. У металевих наночастинках частотна залежність поляризованості визначається в основному плазмовими резонансами. Як відомо, у частинки сферичної форми такий резонанс один, у сфероїдальної форми (еліпсоїд обертання) таких резонансів два, а в еліпсоїдальної частинки (з трьома різними радіусами кривизни) плазмових резонансів три. При відхиленні форми наночастинки від сферичної її поляризованість із скалярної величини стає тензорною величиною. Загальний вигляд тензора поляризованості еліпсоїдальної металевої наночастинки, індукованого дією електромагнітної хвилі, довжина якої значно перевищує розміри частинки, наведено у роботі [1].

За допомогою цього тензора можна виразити переріз поглинання і розсіяння світла металевими наночастинками.
В існуючих теоретичних роботах, де розглядається явище розсіяння світла металевими наночастинками, самі частинки характеризуються такими параметрами, як розмір, їх форма і діелектрична проникність $\epsilon(\omega)$. При цьому вигляд $\epsilon(\omega)$ беремо, як правило, $з$ моделі Друде-Зоммерфельда [1]:

$\epsilon(\omega)=\epsilon^{\prime}(\omega)+i \epsilon^{\prime \prime}(\omega)=1-\frac{\omega_{p}^{2}}{\omega^{2}+\nu^{2}}+i \frac{\nu}{\omega} \frac{\omega_{p}^{2}}{\omega^{2}+\nu^{2}}$,

де $\omega$ - частота світла, $\nu^{-1}$ - час релаксації електронної підсистеми, $\omega_{p}$ - плазмова (об'ємна) частота;

$\omega_{p}=\left(\frac{4 \pi e^{2}}{m} n\right)^{1 / 2}$,

де $e$ i $m$ - відповідно заряд і маса електрона, $n$ - концентрація електронів.

При застосуванні до металевих кластерів формула (1) часто уточнюється. По-перше, замість одиниці у правій частині (1) пишуть $\epsilon_{0}$ - число, яке повинне наближено врахувати внесок іонної підсистеми в $\epsilon(\omega)$. Це число різне для різних металів [3].

По-друге, коли розмір металевої частинки стає меншим за довжину вільного пробігу електрона, то в (1) у випадку сферичної форми частинки відбувається формальна заміна:

$\nu \rightarrow \frac{3}{4} \frac{v_{\mathrm{F}}}{R}$,

де $v_{\mathrm{F}}$ - швидкість Фермі, $R$ - радіус частинки, а в більш загальному вигляді [4]:

$\nu \rightarrow \nu+A \frac{v_{\mathrm{F}}}{R}$

де $A$ - число (ефективний параметр).

Заміна (3) для сферичних наночастинок може бути строго обгрунтована у граничних випадках високих і низьких частот (у порівнянні з частотою зіткнень) $[5,6]$. Проте залишається питання: а як чинити у випадках, коли частинка асиметрична і в одному із напрямків або в усіх напрямках її розміри менші за 
довжину вільного пробігу електрона. Це стає зрозумілим, якщо згадати, що уявна частина діелектричної проникності $\epsilon^{\prime \prime}(\omega)$ у (1) відповідає за процеси дисипації. Зокрема, для розмірів частинки більших довжини вільного пробігу електрона має місце рівність [7]:

$\epsilon^{\prime \prime}(\omega)=\frac{4 \pi}{\omega} \sigma(\omega)$

де $\sigma(\omega)$ - оптична (високочастотна) провідність.

Коли форма металевої частинки асиметрична і їі розмір хоча б в одному напрямку менший за довжину вільного пробігу електрона, то оптична провідність у цьому випадку, як показано у роботі [5], із скаляра стає тензорною величиною. Діагональні елементи цього тензора визначають півширини плазмових резонансів.

Завдання даної роботи полягає у тому, щоб урахувати вплив форми розсіючої світло частинки на процеси розсіяння не тільки через вплив форми на частоти плазмових резонансів, але і на їх півширини. Оскільки півширини визначають висоту плазмових піків, то вплив форми частинки на ці півширини може істотно визначати частотну залежність перерізу розсіяння світла асиметричними металевими наночастинками.

\section{2. Постановка задачі}

Ми починаємо розгляд задачі із загального випадку, коли металева частинка має форму еліпсоїда з трьома радіусами кривизни $\left(R_{x}, R_{y}, R_{z}\right)$. Далі, починаючи 3 певного моменту (вказаного нижче), щоб довести викладки в аналітичному вигляді до кінця, ми виберемо $R_{x}=R_{y} \equiv R_{\perp}, R_{z}=R_{\|}$, тобто обмежимось сфероїдом. Вибір такої форми частинки вигідний тим, що отримані тут аналітичні результати шляхом формальної деформації радіусів кривизни можна перенести на широкий спектр форм наночастинок (від дископодібних до стержнеподібних). Отже, нехай еліпсоїдальна металева наночастинка знаходиться у полі електромагнітної хвилі:

$\mathbf{E}(\mathbf{r}, t)=\mathbf{E}_{0} e^{-i(\omega t-\mathbf{k} \mathbf{r})}$,

де $\mathbf{E}_{0}$ - амплітуда електричного поля хвилі, $\omega$ - 汭 частота, $\mathbf{k}$ - хвильовий вектор, $\mathbf{r}$ - вектор координати, $t$ - час.

Поле (5) індукує всередині металевої частинки внутрішнє електричне поле, яке в дипольному наближенні, тобто при $k R \ll 1$ (де $R=\max \left(R_{x}, R_{y}, R_{z}\right)$ ), зали- шається просторово однорідним і дорівнює [7]:

$E_{\text {in }}^{j} e^{-i \omega t}=\frac{E_{0}^{j} e^{-i \omega t}}{1+L_{j}(\epsilon-1)}$,

де $L_{j}$ - фактор деполяризації.

Внутрішне поле $\mathbf{E}_{\text {in }}$ зумовлюе всередині частинки високочастотний струм, з яким і пов'язана дисипація. Щоб встановити вигляд високочастотного струму і тим самим визначити оптичну провідність металевої наночастинки, потрібно знайти функцію розподілу електронів за швидкостями. Точніше, потрібно знайти доданок до ферміївської функції розподілу, зумовлений дією локального поля (6).

Якщо записати в лінійному наближенні функцію розподілу у вигляді

$f(\mathbf{r}, \boldsymbol{v}, t)=f_{0}(\varepsilon)+f_{1}(\mathbf{r}, \boldsymbol{v}) e^{-i \omega t}$,

де $f_{0}(\varepsilon)$ - ферміївська функція розподілу електронів по енергії $\varepsilon$, то для визначення вигляду $f_{1}(\mathbf{r}, \boldsymbol{v})$ отримуємо кінетичне рівняння

$(\nu-i \omega) f_{1}(\mathbf{r}, \boldsymbol{v})+\boldsymbol{v} \frac{\partial f_{1}(\mathbf{r}, \boldsymbol{v})}{\partial \mathbf{r}}+e \mathbf{E}_{\mathrm{in}} \boldsymbol{v} \frac{\partial f_{0}}{\partial \varepsilon}=0$

Для повного визначення $f_{1}(\mathbf{r}, \boldsymbol{v})$ ця функція, крім рівняння (8), повинна ще задовольняти граничні умови, які визначають характер розсіяння електронів на поверхні металевої частинки. У ролі граничних умов, як і в більшості робіт, приймаємо такі умови, що відповідають дифузному характеру розсіяння, тобто

$\left.f_{1}(\mathbf{r}, \boldsymbol{v})\right|_{s}=0, \quad$ при $\quad v_{n}<0$,

де $v_{n}$ - нормальна до поверхні складова швидкості.

Варто зауважити, що у (8) не враховано дії вихрового електричного поля, зумовленого дією магнітної складової зовнішньої електромагнітної хвилі. Це поле відповідає за так зване магнітне поглинання [5]. $\mathrm{У}$ області частот плазмового резонансу цей механізм поглинання (чи то розсіяння) дає відносно малий внесок $[5,8]$. Граничні умови (9) для випадку еліпсоїдальної форми наночастинки залежать від кутів, і це створює додаткові труднощі при спробі розв'язати рівняння (8). Цих труднощів можна уникнути, якщо у (8) i (9) перейти до деформованої системи координат, в яких еліпсоїдальна форма переходить у сферичну. I так само деформуємо простір швидкостей. Зв'язок деформованих (штрихованих) координат з недеформованими має вигляд

$x_{i}^{\prime}=\gamma_{i} x_{i}, \quad v_{i}^{\prime}=\gamma_{i} v_{i} ; \quad \gamma_{i}=\frac{R}{R_{i}}$, 
$R=\left(R_{x} R_{y} R_{z}\right)^{1 / 3}$

(Для скорочення запису замість $x, y, z$ пишемо $x_{i}$, де $i=1,2,3$, аналогічно і для швидкості $\left.v_{i}\right)$.

Зауважимо, що при деформації (10) об'єм частинки залишається незмінним. Це дозволить далі відокремити ефекти впливу форми від впливу об'єму наночастинки.

У деформованій системі координат рівняння (8) i гранична умова (9) набувають вигляду

$(\nu-i \omega) f_{1}\left(\mathbf{r}^{\prime}, \boldsymbol{v}^{\prime}\right)+\boldsymbol{v}^{\prime} \frac{\partial f_{1}\left(\mathbf{r}^{\prime}, \boldsymbol{v}^{\prime}\right)}{\partial \mathbf{r}^{\prime}}+e \mathbf{E}_{\mathrm{in}} \boldsymbol{v} \frac{\partial f_{0}}{\partial \varepsilon}=0$,

$\left.f_{1}\left(\mathbf{r}^{\prime}, \boldsymbol{v}^{\prime}\right)\right|_{r^{\prime}=R}=0 \quad$ при $\quad \mathbf{r}^{\prime} \boldsymbol{v}^{\prime}<0$.

Рівняння (11) з граничними умовами (12) легко розв'язується методом характеристик $[5,9]$. Як легко переконатись, цей розв'язок виглядає так:

$f_{1}\left(\mathbf{r}^{\prime}, \boldsymbol{v}\right)=-e \mathbf{E}_{\mathrm{in}} \boldsymbol{v} \frac{\partial f_{0}(\varepsilon)}{\partial \varepsilon} \frac{1-e^{-(\nu-i \omega) t_{0}\left(\mathbf{r}^{\prime}, \boldsymbol{v}^{\prime}\right)}}{\nu-i \omega}$,

де характеристика $t_{0}\left(\mathbf{r}^{\prime}, \boldsymbol{v}^{\prime}\right)$ має вигляд

$t_{0}\left(\mathbf{r}^{\prime}, \boldsymbol{v}^{\prime}\right)=\frac{1}{v^{\prime 2}}\left\{\mathbf{r}^{\prime} \boldsymbol{v}^{\prime}+\sqrt{\left(R^{2}-r^{\prime 2}\right) v^{\prime 2}+\left(\mathbf{r}^{\prime} \boldsymbol{v}^{\prime}\right)^{2}}\right\}$

Використовуючи функцію (13), можна визначити густину високочастотного струму у металевій наночастинці:

$\mathbf{I}(\mathbf{r}, \omega)=2 e\left(\frac{m}{2 \pi \hbar}\right)^{3} \iiint \boldsymbol{v} f_{1}(\mathbf{r}, \boldsymbol{v}) d^{3} v$.

Якщо ввести тензор комплексної провідності $\sigma_{\alpha \beta}^{c}(\mathbf{r}, \omega)$ згідно зі співвідношенням

$I_{\alpha}(\mathbf{r}, \omega)=\sum_{\beta=1}^{3} \sigma_{\alpha \beta}^{c}(\mathbf{r}, \omega) E_{\mathrm{in}}^{\beta}$,

то для компонент цього тензора з (13) і (15) отримуEMO

$\sigma_{\alpha \beta}^{c}(\mathbf{r}, \omega)=2 e\left(\frac{m}{2 \pi \hbar}\right)^{3} \times$

$\times \iiint v_{\alpha}\left\{-e v_{\beta} \frac{\partial f_{0}}{\partial \varepsilon} \frac{1-e^{-(\nu-i \omega) t_{0}\left(\mathbf{r}^{\prime}, \boldsymbol{v}^{\prime}\right)}}{\nu-i \omega}\right\} d^{3} v$.

Тут варто наголосити, що хоча поле $E_{\text {in }}$ всередині еліпсоїдальної частинки в дипольному наближенні не залежить від координат, функція розподілу $f_{1}(\mathbf{r}, \boldsymbol{v})$ стає залежною від координат завдяки граничній умові (12), а тому і густина струму $I(\mathbf{r}, \omega)$, і комплексна провідність $\sigma_{\alpha \beta}^{c}(\mathbf{r}, \omega)$ стають залежними від координат. Проте фізичний зміст мають, як правило, усереднені по об'єму наночастинки величини. Зокрема енергія, яка за одиницю часу поглинається металевою наночастинкою у результаті дії на неї монохроматичної хвилі (5), має вигляд

$w=\frac{1}{2} \operatorname{Re} \int d \mathbf{r}\left\{\mathbf{I}\left(\mathbf{r}^{\prime}, \omega\right) \mathbf{E}_{\text {in }}^{*}\right\}=$

$=\frac{V}{2} \sum_{\alpha, \beta=1}^{3} R_{e}\left\langle\sigma_{\alpha \beta}^{c}(\omega)\right\rangle E_{\mathrm{in}}^{\alpha}\left(E_{\mathrm{in}}^{\beta}\right)^{*}$,

де

$\left\langle\sigma_{\alpha \beta}^{c}(\omega)\right\rangle=\frac{1}{V} \int d \mathbf{r}^{\prime} \sigma_{\alpha \beta}^{c}\left(\mathbf{r}^{\prime}, \omega\right)$

- всереднені по об'єму наночастинки $(V)$ компоненти тензора комплексної провідності.

Між компонентами тензора діелектричної проникності існує відомий зв'язок [10]:

$\epsilon_{\alpha \beta}(\mathbf{r}, \omega)=\delta_{\alpha \beta}+i \frac{4 \pi}{\omega} \sigma_{\alpha \beta}^{c}(\mathbf{r}, \omega)$.

Вплив поверхні на явище провідності описується в (17) характеристикою $t_{0}\left(\mathbf{r}^{\prime}, \boldsymbol{v}^{\prime}\right)$. Як видно із (14), по порядку величини $t_{0} \sim R / v_{\mathrm{F}}$. Це означає, що по порядку величини $t_{0}^{-1} \sim v_{\mathrm{F}} / R$ відповідає прольотній частоті (електрона від стінки до стінки). Тому нерівність $\nu t_{0} \gg 1$ означала б, що об'ємна частота зіткнень $\nu$ значно більша за частоту зіткнень електрона $з$ поверхнею. У цьому випадку, приймаючи формально $\nu \rightarrow \infty$ i, крім того, $\partial f_{0} / \partial \varepsilon \sim-\delta\left(\varepsilon-\varepsilon_{\mathrm{F}}\right)$ із (17), отримуємо

$\sigma_{\alpha \beta}(\omega)=\frac{1}{4 \pi} \frac{\omega_{p}^{2}}{\nu-i \omega} \delta_{\alpha \beta}$

Як видно із (21), провідність у данному випадку залишається скалярною величиною і не залежить від координат. Якщо підставити (21) у (20), то отримаємо вираз для діелектричної проникності у вигляді (1).

У загальному випадку еліпсоїдальної форми металевої наночастинки, меншої за розміром довжини вільного пробігу електрона (коли істотне розсіяння електронів на поверхні), оптична провідність (17) 
має тензорний характер. Завдяки цьому і комплексна діелектрична проникність наночастинки також стає тензором. У цьому випадку вимагає уточнення і зв'язок внутрішнього поля $\mathbf{E}_{\text {in }}$ з електричним полем зовнішньої електромагнітної хвилі $\mathbf{E}_{0}$. Замість (6) у головних осях еліпсоїда цей зв'язок буде мати вигляд

$E_{\mathrm{in}}^{j} e^{-i \omega t}=\frac{E_{0}^{\beta} e^{-i \omega t}}{1+L_{\beta}\left(\epsilon_{\beta \beta}(\omega)-1\right)}$,

де $\epsilon_{\beta \beta}(\omega)$ - діагональна компонента тензора діелектричної проникності (у напрямку осі $\beta ; \epsilon_{\beta \beta}(\omega) \equiv$ $\left.\left\langle\epsilon_{\beta \beta}(\omega)\right\rangle\right)$.

\section{3. Тензор поляризованості}

Індукований полем електромагнітної хвилі електричний дипольний момент у металевій наночастинці, пов'язаний з високочастотним струмом $\mathbf{I}(\mathbf{r}, t)$ співвідношенням

$\frac{\partial}{\partial t} \mathbf{d}(t)=\int_{V} d \mathbf{r} \mathbf{I}\left(\mathbf{r}^{\prime}, t\right)$

Якщо у (23) зробити перетворення Фур'є і використати (16), то отримаємо

$\underset{\alpha}{d}(\omega)=\frac{i V}{\omega} \sum_{\beta=1}^{3}\left\langle\sigma_{\alpha \beta}^{c}(\omega)\right\rangle E_{\mathrm{in}}^{\beta}$.

Далі введемо тензор поляризованості співвідношенням

$\underset{\beta}{d}(\omega)=\sum_{\beta^{\prime}=1}^{3} \alpha_{\beta \beta^{\prime}}(\omega) E_{0}^{\beta^{\prime}}$.

Порівнюючи між собою вирази (24), (25) і використовуючи (22), маємо

$\alpha_{\beta \beta}(\omega)=\frac{i V}{\omega} \frac{\left\langle\sigma_{\beta \beta}^{c}(\omega)\right\rangle}{1+L_{\beta}\left(\epsilon_{\beta \beta}(\omega)-1\right)}$.

Якщо тепер застосувати співвідношення (20) (провівши попередньо усереднення його по об'єму частинки), то із (26) випливає

$\alpha_{\beta \beta}(\omega)=\frac{V}{4 \pi} \frac{\epsilon_{\beta \beta}(\omega)-1}{1+L_{\beta}\left(\epsilon_{\beta \beta}(\omega)-1\right)}$.

Тепер бачимо, що знайшовши із (17) компоненти тензора комплексної провідності, за допомогою (20) i
(27) легко отримаємо тензор поляризованості металевої наночастинки. Згідно із (17) усереднене значення компонент тензора комплексної провідності має вигляд

$$
\begin{aligned}
& \left\langle\sigma_{\alpha \beta}^{c}\right\rangle=\left(\frac{m}{2 \pi \hbar}\right)^{3} \frac{2 e^{2}}{\nu-i \omega} \int \frac{d^{3} r^{\prime}}{V} \times \\
& \times \int d^{3} v v_{\alpha} v_{\beta} \delta\left(\varepsilon-\varepsilon_{\mathrm{F}}\right)\left\{1-e^{-(\nu-i \omega) t_{0}\left(\mathbf{r}^{\prime}, \boldsymbol{v}^{\prime}\right)}\right\} .
\end{aligned}
$$

Якщо тепер використати значення інтеграла (деталі див. $[6,9])$

$\int \frac{d^{3} r^{\prime}}{V}\left\{1-e^{-(\nu-i \omega) t_{0}\left(\mathbf{r}^{\prime}, \boldsymbol{v}^{\prime}\right)}\right\}=$

$=\frac{3}{4}\left\{\frac{4}{3}-\frac{2}{q}+\frac{4}{q^{3}}-\frac{4}{q^{2}}\left(1+\frac{1}{q}\right) e^{-q}\right\} \equiv \frac{3}{4} \Psi(q)$,

де $q=\frac{2}{v^{\prime}}(\nu-i \omega)$, то для компонент тензора провідності (28) отримаємо вираз

$\left\langle\sigma_{\alpha \beta}^{c}(\omega)\right\rangle=\frac{3}{2}\left(\frac{m}{2 \pi \hbar}\right)^{3} \frac{e^{2}}{\nu-i \omega} \times$

$\times \int \frac{d^{3} r^{\prime}}{V} \int d^{3} v \delta\left(\varepsilon-\varepsilon_{\mathrm{F}}\right) v_{\alpha} v_{\beta} \Psi(q)$.

Усереднені значення компонент тензора комплексної провідності (29), як і всі попередні викладки, отримані для загального випадку еліпсоїдальної форми наночастинки з трьома різними радіусами кривизни $\left(R_{x}, R_{y}, R_{z}\right)$. Подальші обчислення кутових інтегралів у формулі (29) ускладнюються тим, що модуль деформованого вектора швидкості $v^{\prime}$ у виразі $q=q\left(v^{\prime}\right)$ залежить від кутів відповідно до (10). Значно простіше ця залежність виглядає у випадку еліпсоїда обертання (коли $R_{x}=R_{y}=R_{\perp} ; R_{z} \equiv R_{||}$). Відповідно до (10) у цій ситуації

$v^{\prime}=R\left\{\frac{\sin ^{2} \theta}{R_{\perp}^{2}}+\frac{\cos ^{2} \theta}{R_{\|}^{2}}\right\}^{1 / 2} v$,

де $\theta$ - кут між вектором $v$ і віссю обертання еліпсоїда.

Подальші обчислення $\left\langle\sigma_{\alpha \beta}^{c}(\omega)\right\rangle$ згідно із $(29)$ не викликають труднощів (див. [5]).

Для сфероїдальної форми металевої наночастинки, як можна бачити із формул (29), (27) і (20), відмінними від нуля будуть компоненти тензора поляризованості:

$\alpha_{x x}=\alpha_{y y} \equiv \alpha_{\perp} ; \quad \alpha_{z z}=\alpha_{\perp}$. 
При цьому відповідно до (21) маємо

$\alpha_{\perp, \|}(\omega)=\frac{V}{4 \pi} \frac{\epsilon_{\perp, \|}(\omega)-1}{1+L_{\perp, \|}\left(\epsilon_{\perp, \|}(\omega)-1\right)}$,

де для $\epsilon_{\perp, \|}(\omega)$ iз $(29)$ і (20) отримуємо

$\epsilon_{\perp, \|}(\omega)=1-\frac{\omega_{p}^{2}}{\omega^{2}}+i \frac{4 \pi}{\omega} \sigma_{\perp, \|}(\omega)$.

У (33) прийнято позначення:

$\sigma_{\perp}(\omega)=\operatorname{Re}\left\langle\sigma_{x x}^{c}(\omega)\right\rangle=\operatorname{Re}\left\langle\sigma_{y y}^{c}(\omega)\right\rangle$

$\sigma_{\|}(\omega)=\operatorname{Re}\left\langle\sigma_{z z}^{c}(\omega)\right\rangle$

Нас цікавить розсіяння світла малими частинками у видимому діапазоні частот. Тому при отриманні (33) iз (20) i (29) ми нехтували малими поправками, пропорційними відношенню частоти зіткнень до частоти світла. У цьому ж наближенні із (29) для наночастинок, менших за розміром довжини вільного пробігу електрона, маємо [5]:

$\sigma_{\perp}(\omega)=\frac{9}{16} \frac{n e^{2} v_{\mathrm{F}}}{m \omega^{2}}\left\{\begin{array}{cll}\frac{3 \pi}{8 R_{\perp}}, & \text { при } R_{\perp} \ll R_{\|} \\ \frac{1}{2 R_{\|}}, & \text {при } R_{\perp} \gg R_{\|}\end{array}\right\}$,

$\sigma_{\|}(\omega)=\frac{9}{16} \frac{n e^{2} v_{\mathrm{F}}}{m \omega^{2}}\left\{\begin{array}{ccc}\frac{\pi}{4 R_{\perp}}, & \text { при } R_{\perp} \ll R_{\|} \\ \frac{1}{R_{\|}}, & \text {при } R_{\perp} \gg R_{\|}\end{array}\right\}$.

Крім того, при $R_{\perp}=R_{\|}=R$ :

$\sigma_{\perp}(\omega)=\sigma_{\|}(\omega)=\frac{3}{4} \frac{n e^{2} v_{\mathrm{F}}}{m \omega^{2} R}$.

Отже, формули (32), (33) та (35)-(37) повністю визначають залежності від форми компонент тензора поляризованості. Ще раз нагадуємо, що хоча частинка має форму сфероїда, співвідношення між радіусами кривизни $R_{\perp}$ і $R_{\|}$може бути довільним (при фіксованому об'ємі частинки див. (10)). Точний вираз для $\sigma_{\perp, \|}(\omega)$ при довільних $R_{\perp}$ і $R_{\|}$наведено у $[5,11]$.

\section{4. Переріз розсіяння світла металевими наночастинками}

Індукований електричною складовою електромагнітної хвилі (5) дипольний момент у металевій наночастинці, у свою чергу, генерує розсіяну хвилю. Крім електричного дипольного моменту може генеруватись (магнітною складовою хвилі) також магнітний дипольний момент. Вище вже було пояснено, чому ми його не розглядаємо. Будемо розглядати розсіяну хвилю на далекій відстані від частинки (порівняно $з$ довжиною хвилі), де вже розсіяну хвилю можна вважати поперечною. Електричну складову розсіяної хвилі будемо позначати через $\mathbf{E}^{\prime}$, а магнітну - через $\mathbf{H}^{\prime}$ (при цьому $E^{\prime}=H^{\prime}$ ). Середня (за період) інтенсивність випромінювання на відстані $R_{0}$ в тілесний кут $d \Omega$, генерованого електричним диполем, буде [7]:

$d I_{s}=\frac{c}{8 \pi}\left|\left[\mathbf{E}^{\prime} \times \mathbf{H}^{\prime}\right]\right| R_{0}^{2} d \Omega=\frac{c}{8 \pi}\left|\mathbf{H}^{\prime}\right|^{2} R_{0}^{2} d \Omega$,

де $c$ - швидкість світла.

Оскільки при випромінюванні електричного диполя маємо [7]:

$\mathbf{H}^{\prime}=\frac{\omega^{2}}{c^{2} R_{0}} \mathbf{n}_{0} \times \mathbf{d}(\omega)$,

де $\mathbf{n}_{0}-$ орт, який визначає напрямок спостереження, то для перерізу розсіяння отримуємо вираз

$d \Sigma=d I_{s} / \frac{c}{8 \pi}\left|\mathbf{H}^{\prime}\right|^{2}$.

Кутова залежність інтенсивності розсіяного сфероїдальною частинкою світла визначається, крім введеного вище орту $\mathbf{n}_{0}$, ще двома ортами: $\mathbf{p}_{0}-$ орт, який задає поляризацію вихідного світлового потоку і $\mathbf{q}_{0}-$ орт, який дає орієнтацію осі симетрії сфероїда (див. рисунок).

У головних осях сфероїда, який видно із (31) і (25), мaємо

$\mathbf{d}(\omega)=\alpha_{\perp}(\omega) \mathbf{E}_{0}^{\perp}+\alpha_{\|}(\omega) \mathbf{E}_{0}^{\|}$,

де

$\mathbf{E}^{\|}=\left(\mathbf{q}_{0} \mathbf{E}_{0}\right) \mathbf{q}_{0} ; \quad \mathbf{E}_{0}^{\perp}=\mathbf{E}_{0}-\left(\mathbf{q}_{0} \mathbf{E}_{0}\right) \mathbf{q}_{0}$.

Як бачимо, дипольний момент сфероїдальної частинки складається із суми двох взаємно перпендикулярних моментів.

Використовуючи (39) і (41), отримуємо

$\left|\mathbf{H}^{\prime}\right|^{2}=\frac{\omega^{4}}{c^{4} R_{0}^{2}}\left\{\left|\alpha_{\perp}(\omega)\right|^{2}\left(\mathbf{n}_{0} \times \mathbf{E}_{0}^{\perp}\right)^{2}+\right.$

$+\left|\alpha_{\|}(\omega)\right|^{2}\left(\mathbf{n}_{0} \times \mathbf{E}_{0}^{\|}\right)^{2}+\left(\alpha_{\perp}(\omega) \alpha_{\|}^{*}(\omega)+\alpha_{\perp}^{*}(\omega) \alpha_{\|}(\omega)\right) \times$

$\left.\times\left(\mathbf{n}_{0} \times \mathbf{E}_{0}^{\perp}\right)\left(\mathbf{n}_{0} \times \mathbf{E}_{0}^{\|}\right)\right\}$. 


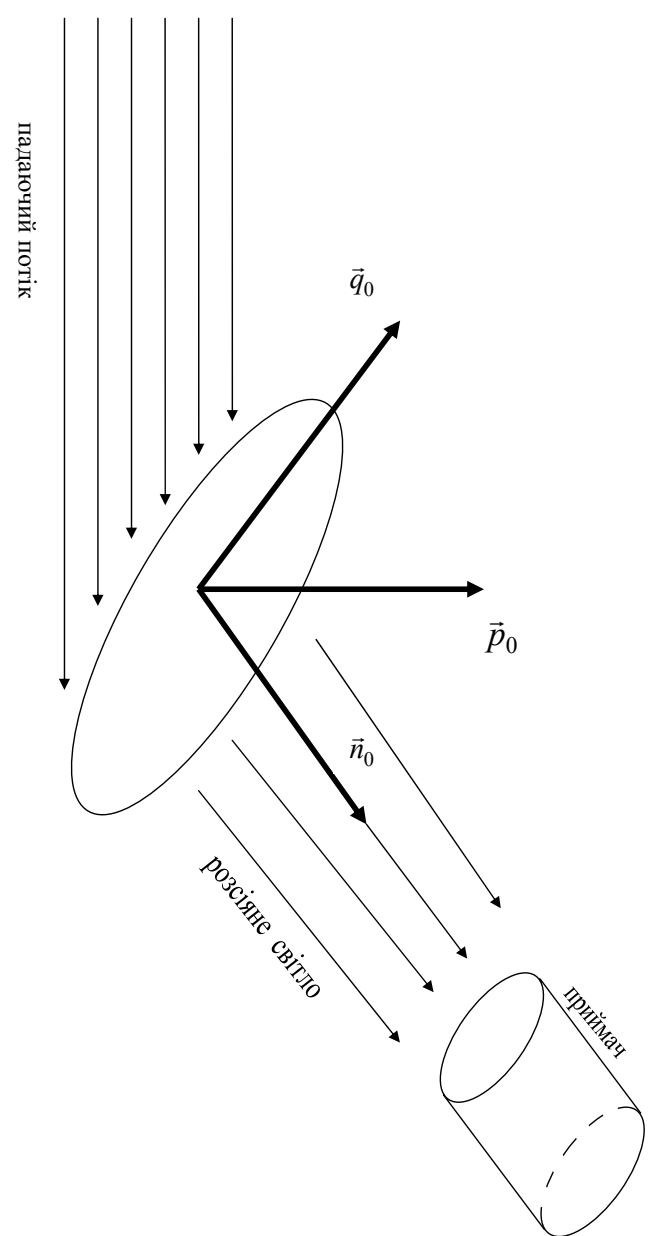

Схема розсіяння (зображена тільки та частина розсіяного потоку, яка спрямована у напрямку приймача

Підставимо (43) у (38) i, використовуючи (40) i (42), отримуємо для перерізу розсіяння вираз

$$
\begin{aligned}
& d \Sigma=\frac{\omega^{4}}{c^{4}}\left\{\left|\alpha_{\perp}(\omega)\right|^{2}\left[\mathbf{n}_{0} \times\left(\mathbf{p}_{0}-\left(\mathbf{q}_{0} \mathbf{p}_{0}\right) \mathbf{q}_{0}\right)\right]^{2}+\right. \\
& +\left|\alpha_{\|}(\omega)\right|^{2}\left(\mathbf{n}_{0} \times \mathbf{q}_{0}\right)^{2}\left(\mathbf{q}_{0} \mathbf{p}_{0}\right)^{2}+2 \operatorname{Re} \alpha_{\perp}(\omega) \times
\end{aligned}
$$$$
\left.\times \alpha_{\|}^{*}(\omega)\left[\mathbf{n}_{0} \times\left(\mathbf{p}_{0}-\left(\mathbf{q}_{0} \mathbf{p}_{0}\right) \mathbf{q}_{0}\right)\right]\left(\mathbf{n}_{0} \times \mathbf{q}_{0}\right)\left(\mathbf{q}_{0} \mathbf{p}_{0}\right)\right\} d \Omega .
$$

У випадку сферичної форми $\left(\alpha_{\perp}=\alpha_{\|}=\alpha\right)$ всі члени в (44), які містять орт q0, як не складно побачити, взаємно скорочуються, і ми отримуємо відомий для сферичної частинки вираз

$d \Sigma=\frac{\omega^{4}}{c^{4}}|\alpha(\omega)|^{2}\left(\mathbf{n}_{0} \times \mathbf{p}_{0}\right)^{2} d \underline{0}=\frac{\omega^{4}}{c^{4}}|\alpha(\omega)|^{2} \sin ^{2} \nu^{\prime} d \underline{0}$, де $\nu^{\prime}-$ кут між $\mathbf{n}_{0}$ і електричним полем $\mathbf{E}_{0}$.

Оскільки в літературі (щоб акцентувати увагу на ролі об'єму частинки у процесі розсіяння) часто використовують питому поляризованість, то нагадаємо, що у цій роботі згідно із (32) мова йде про поляризованість всієї частинки.

Отже, вище ми у найпростішому випадку отримали вираз (44) для перерізу розсіяння світла сфероїдальною металевою частинкою. Щоб уникнути непорозумінь, ще раз нагадаємо, що загальні вирази для перерізів поглинання і розсіяння світла через діагональні елементи тензора поляризованості еліпсоїдальної металевої частинки наведено, наприклад, у монографії [1]. Там же показано і усереднені значення цих величин при хаотичній орієнтації однакових еліпсоїдальних частинок. Проте там і в інших роботах, (див. також [12]) для діелектричної проникності використовують вираз, який відповідає моделі ДрудеЗоммерфельда, тобто вираз (1). Як вже наголошувалось вище, модель Друде-Зоммерфельда потребує певної модифікації, коли розміри наночастинки стають меншими за довжину вільного пробігу електрона [11] . Суть цієї модифікації стає зрозумілою з огляду на вирази (32) і (33). Зокрема із (32) і (33) отримуємо

$\left|\alpha_{\perp, \|}(\omega)\right|^{2}=\left(\frac{V}{4 \pi}\right)^{2} \frac{\omega_{p}^{4}+\left(4 \pi \sigma_{\perp, \|}(\omega) \omega\right)^{2}}{\left(\omega^{2}-\omega_{\perp, \|}^{2}\right)^{2}+\left(4 \pi L_{\perp, \|} \sigma_{\perp, \|}(\omega) \omega\right)^{2}}$,

де

$\omega_{\perp, \|} \equiv \sqrt{L_{\perp, \|}} \omega_{p}$

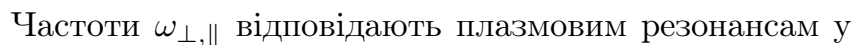
сфероїдальних металевих частинках. Другим членом у чисельнику можна нехтувати у видимому діапазоні частот (у порівнянні з $\omega_{p}^{4}$ ). Як видно із $(46)$, діагональні компоненти тензора поляризованості мають різкі піки на частотах плазмових резонансів. Півширини цих піків визначаються діагональними елементами тензора оптичної провідності $\sigma_{\perp, \|}(\omega)$. Зокрема, відношення висот цих піків, як видно із (46), буде таким:

$\left|\alpha_{\perp}(\omega)\right|_{\omega=\omega_{\perp}}^{2} /\left|\alpha_{\|}(\omega)\right|_{\omega=\omega_{\|}}^{2} \approx\left\{\frac{L_{\|} \sigma_{\|}\left(\omega_{\|}\right) \omega_{\|}}{L_{\perp} \sigma_{\perp}\left(\omega_{\perp}\right) \omega_{\perp}}\right\}^{2}$.

Залежність виразів (46) і (48) від форми частинки закладена як у коефіцієнтах деполяризації $\left(L_{\perp}, L_{\|}\right)$, так і у компонентах тензора оптичної провідності $\left(\sigma_{\perp}(\omega), \sigma_{\|}(\omega)\right)$. 
Щодо розмірної залежності компонент тензора оптичної провідності сфероїдальної металевої частинки, то для різних граничних випадків вони задаються формулами (35)-(37). Загальний же вигляд цих компонент наведено у наших роботах $[5,6,11]$.

Коефіцієнти деполяризації у випадку еліпсоїда обертання мають простий вигляд [7]:

$L_{x}=L_{y} \equiv L_{\perp}=\frac{1}{2}\left(1-L_{\|}\right)$

$L_{z} \equiv L_{\|}=\left\{\begin{array}{ll}\frac{1-e_{p}^{2}}{2 e_{p}^{3}}\left(\ln \frac{1+e_{p}}{1-e_{p}}-2 e_{p}\right), & R_{\perp}<R_{\|} \\ \frac{1+e_{p}^{2}}{e_{p}^{3}}\left(e_{p}-\arctan e_{p}\right), & R_{\perp}>R_{\|}\end{array}\right\}$

де

$e_{p}^{2} \equiv\left|1-R_{\perp}^{2} / R_{\|}^{2}\right|$

Легко бачити, що вираз (37) для високочастотної провідності у сферичному випадку $\left(R_{\perp}=R_{\|}=\right.$ $R$ ) можна отримати 3 (1), якщо врахувати (4) i підстановку (3). Нагадаємо, що така підстановка широко використовується у літературі для розмірів наночастинок, менших за довжину вільного пробігу електрона. Проте, в асиметричному випадку $\left(R_{\perp} \neq R_{\|}\right)$спроба використати підстановку (3) з якимось проміжним значенням $R$ (між мінімальним і максимальним розмірами наночастинки) не дає бажаного результату. Як видно із (48) i (35)-(37), під час використання такої апроксимації і при використанні справжніх компонент тензора провідності (35), (36) відмінність по висоті плазмових піків буде у декілька разів. Наприклад, у випадку сплюснутого сфероїда $\left(R_{\perp} \gg R_{\|}\right)$отримані результати будуть відрізнятись у чотири рази.

Отже, обчислення впливу форми наночастинки на півширини плазмових резонансів у задачах розсіяння світла є принципово важливим і не зводиться до малих поправок відомих результатів для сферичної частинки.

Вище ми на прикладі виразу $\left|\alpha_{\perp, \|}(\omega)\right|^{2}$, який входить у формулу для перерізу розсіяння (44), коротко проаналізували вплив форми наночастинки на процеси розсіяння світла. Крім $\left|\alpha_{\perp, \|}(\omega)\right|^{2}$ в (44) залежним від форми частинки входить також вираз $\operatorname{Re}\left(\alpha_{\perp}(\omega) \alpha_{\|}^{*}(\omega)\right)$. Цей вираз трохи менше чутливий до форми розсіючої частинки, і ми на ньому зупинятися не будемо.

\section{5. Висновки}

$\mathrm{Y}$ роботі отримано аналітичний вираз для перерізу розсіяння світла металевою частинкою сфероїдальної форми, в якому враховано не тільки вплив форми частинки на частоти плазмових резонансів (що враховувалось і раніше), але і вплив цієї форми на півширини плазмових резонансів. У випадку розмірів наночастинки, менших за довжину вільного пробігу електрона, провідність асиметричної частинки стає тензорною величиною, і діагональні елементи цього тензора визначають (разом з коефіцієнтами деполяризації) півширини плазмових резонансів. А вже плазмові резонанси і їх півширини визначають інтенсивність розсіяння світла у діапазоні частот близьких до резонансів. Показано, що результати для перерізу розсіяння світла анізотропною металевою наночастинкою, отримані з урахуванням і без урахування впливу форми наночастинки на півширини плазмових резонансів, можуть відрізнятися у декілька разів.

1. В.В. Климов, Плазмоника (Физматлит, Москва, 2010).

2. Є.Ф. Венгер, А.В. Гончаренко, М.Л. Дмитрук, Оптика малих частинок $і$ дисперсійних середовищ (Наукова думка, Київ, 1999).

3. P. Johnson and R. Christy, Phys. Rev. B 6, 4370 (1972).

4. L. Genzel and U. Kreibig, Z. Phys. B 37, 93 (1980).

5. P.M. Tomchuk and N.I. Grigorchuk, Phys. Rev. B 73, 155423 (2006).

6. Н.И. Григорчук, П.М. Томчук, ФНТ, 33, 461 (2007).

7. Л.Д. Ландау, Е.М. Лифшиц, Электродинамика сплошных сред (Изд. Технико-теоретической литературы, Москва, 1957).

8. N.I. Grigorchuk and P.M. Tomchuk, Phys. Rev. B 80, 155456 (2009).

9. А.Г. Лескис, В.Е. Пастернак, А.А. Юшканов, ЖЭТФ bf 83,310 (1982).

10. А.Ф. Александров, Л.С. Богданкевич, А.А. Рухадзе, Основы электродинамики плазмы (Высшая школа, Москва, 1978).

11. N.I. Grigorchuk and P.M. Tomchuk, Phys. Rev. B 84, 085448 (2011).

12. В.В. Климов, УФН 178, 0875 (2008).

Одержано 21.09.11 


\section{ЗАВИСИМОСТЬ СЕЧЕНИЯ РАССЕЯНИЯ СВЕТА МЕТАЛЛИЧЕСКИМИ НАНОЧАСТИЦАМИ ОТ ИХ ФОРМЫ}

\section{П.М. Томчук}

$\mathrm{P}$ е $з$ ю м

Построена теория рассеяния света эллипсоидальными частицами, размеры которых меньше длины свободного пробега электрона, а также значительно меньше длины электромагнитной волны. Учтено влияние формы наночастицы как на частоты плазменных резонансов, так и на их полуширины. В задаче рассеяния света впервые учтено тензорный характер оптической проводимости, что свойственно для эллипсоподобных металлических наночастиц таких размеров. Полученное выражение для сечения рассеяния иллюстрирует существенное влияние формы частицы на частотные и поляризационные зависимости рассеянного света.

\section{DEPENDENCE OF LIGHT SCATTERING CROSS-SECTION} BY METAL NANOPARTICLES ON THEIR SHAPE

\section{P.M. Tomchuk}

Institute of Physics, Nat. Acad. of Sci. of Ukraine

(46, Nauky Ave., Kyiv 03680, Ukraine;

e-mail: ptomchuk@iop.kiev.ua)

$\mathrm{S} \mathrm{u} \mathrm{m} \mathrm{m} \mathrm{a} \mathrm{r} \mathrm{y}$

A theory of light scattering by ellipsoidal metal nanoparticles, the size of which is smaller than the free electron path length in those nanoparticles and much smaller than the light wavelength, has been developed. Effects of nanoparticle shape on both the frequencies and the band half-widths of plasmon resonances have been taken into account. The tensor character of the optical conductivity, which is typical of ellipsoidal metal nanoparticles with such dimensions, has been considered for the first time in the framework of the light scattering problem. The obtained formula for the scattering cross-section demonstrates a substantial influence of the nanoparticle shape on the frequency and polarization dependences of scattered light. 